\title{
Design of simulated moving bed and Varicol processes for preparative separations with a low number of columns
}

\author{
Luís S. Pais ${ }^{\mathrm{a}, \mathrm{b}}$, Alírio E. Rodrigues ${ }^{\mathrm{a}, *}$ \\ ${ }^{a}$ Laboratory of Separation and Reaction Engineering, School of Engineering, University of Porto, Rua Dr. Roberto Frias s/n, \\ 4200-465 Porto, Portugal \\ ${ }^{\mathrm{b}}$ School of Technology and Management, Bragança Polytechnic Institute, Campus de Santa Apolónia, Apartado 1134, \\ 5301-857 Bragança, Portugal
}

\begin{abstract}
Simulated moving bed (SMB) chromatography has received significant attention in the last decade, particularly as regards the production of very valuable products, such as enantiomerically pure pharmaceutical compounds. Recent applications in the pharmaceutical industry use SMB systems containing a low total number chromatographic columns, usually four to eight. This paper deals with the modeling and simulation of SMB systems with only four, five and six columns. In particular, two modeling strategies, the equivalent true moving bed and the real SMB models, are compared for these units in terms of separation regions and system productivity. Also, the recently proposed Varicol process is analyzed and compared with the classical SMB operation, and the advantages of this new operation mode are shown for systems using a low number of columns.
\end{abstract}

(C) 2003 Elsevier B.V. All rights reserved.

Keywords: Simulated moving bed chromatography; Varicol process; Preparative chromatography; Enantiomer separation; Computer simulation

\section{Introduction}

Simulated moving bed (SMB) chromatography is a continuous chromatographic process that overcomes the usual limitations of standard preparative chromatographic methods, namely the high inventories of solvent and adsorbent needed and the high dilution of products. SMB technology, which has been used for decades in large-scale separations in the petrochemical and carbohydrate industries, has recently found new successful applications in the

\footnotetext{
*Corresponding author. Tel.: +351-22-508-1671; fax: +35122-508-1674.

E-mail address: arodrig@fe.up.pt (A.E. Rodrigues).
}

areas of biotechnology, pharmaceuticals and fine chemistry.

The first publications addressing the use of SMB technology for the production of pure enantiomers appeared in 1992 and, 10 years later a considerable number of studies have been developed and reported by the industry and academia, concerning the experimental operation of SMB units for chiral separations [1-28]. Several pharmaceutical companies and custom chemical manufactures are installing commercial-scale SMB units for producing enantiomeric compounds. The list is rapidly increasing and includes Aerojet Fine Chemicals (USA), Bayer (Germany), CarboGen Laboratories (Switzerland), Chiral Technologies (USA), Daicel (Japan), Honeywell Specialty Chemicals (Ireland), H. Lundbeck (Den- 
mark), Merck (Germany), UCB Pharma (Belgium), and Universal Pharma Technologies (USA) [29-32].

Modeling and simulation of a chemical engineering process has always attracted special attention since it could lead to significant savings in time and materials. The selection of SMB operating conditions is not straightforward and requires the use of modeling and simulation tools. The main problem with the SMB operation consists of choosing the right solid (switch time interval) and liquid flow-rates. Designed for high productivity separations, SMB units usually operate at high feed concentrations leading to nonlinear competitive adsorption behaviors. Therefore, modeling and simulation tools are of crucial importance before running the system.

Two models are frequently used to simulate an SMB unit: one is the true moving bed (TMB) model, which assumes equivalence with an ideal unit where the solid and the liquid phases move counter-currently; the other is the real SMB model, where the dynamics associated with the periodic shift of the inlet and outlet lines are taken into account. In a recent paper [33], we concluded that prediction of the SMB operation can be carried out through the equivalent TMB approach when the SMB unit is constituted by, at least, two columns per section (a total of eight columns). However, recent applications in the pharmaceutical industry use SMB systems containing a low total number of columns. According to Nicoud and Majors [34], for pharmaceutical applications, the number of columns tends to be a maximum of 12 for pilot-plant uses and a maximum of eight for industrial applications. Lehoucq et al. [22] reported the experimental operation of an SMB unit for the separation of a racemic mixture using only six chromatographic columns. Novasep (Vandoeuvre Les Nancy, France), a leading supplier of SMB industrial units, has announced the installation of the largest unit for chiral separations with only six columns [35]. In view of these new trends, it is important to study the performance of SMB systems with a low number of columns and to compare the two strategies of modeling, TMB and SMB, for these cases. This is the first objective of this work.

Additionally, Novasep proposed recently a new continuous chromatographic process, called Varicol [36,37]. The principle of the Varicol process is based on a non-synchronous shift of the inlet and outlet valves in a multicolumn system, in contrast to the SMB operation where this shift is synchronous. This new process makes possible operation with a number of columns per section that is not constant in time, and can show advantages over the classical SMB operation, particularly when using a low number of columns. The second objective of this work is to evaluate and compare the performance of SMB and Varicol systems with a low number of columns. Simulation results will be shown for units with four, five, and six columns.

\section{SMB strategies of modeling}

The problem of modeling an SMB separation process can be analyzed by two different strategies: firstly, by simulating the system directly, taking into account its intermittent behavior, and secondly by representing its operation in terms of a true countercurrent system. The first model represents the real SMB and considers the periodic switch of the injection and collection points. The second is developed by assuming equivalence with the true moving bed (TMB), where solid and fluid phases flow in opposite directions (Fig. 1).

The model equations used in this work result from the mass balance over a volume element of the bed and at a particle level. Axial dispersion flow for the bulk fluid phase is included and the linear driving force approximation is used to describe the intraparticle mass-transfer rate; the model can handle any kind of adsorption isotherm. The model equations for the transient SMB model are summarized in Table 1, Eqs. (1a) and (1b), where the subscript $i$ refers to the component, and $k$ refers to the chromatographic column. To complete the modeling of the SMB system, initial and boundary conditions, mass balances at the nodes between each column, and a model for the adsorption equilibrium isotherms must be added (Table 1, Eqs. (2)-(6)). Due to the switch of inlet and outlet lines, each column plays different functions during a whole cycle, depending on its location. In this way, for the SMB model, the boundary conditions for each column change after the end of each switch time interval. This timedependence of the boundary conditions leads to a cyclic steady state for this system, instead of a real 


\section{(a) SMB}

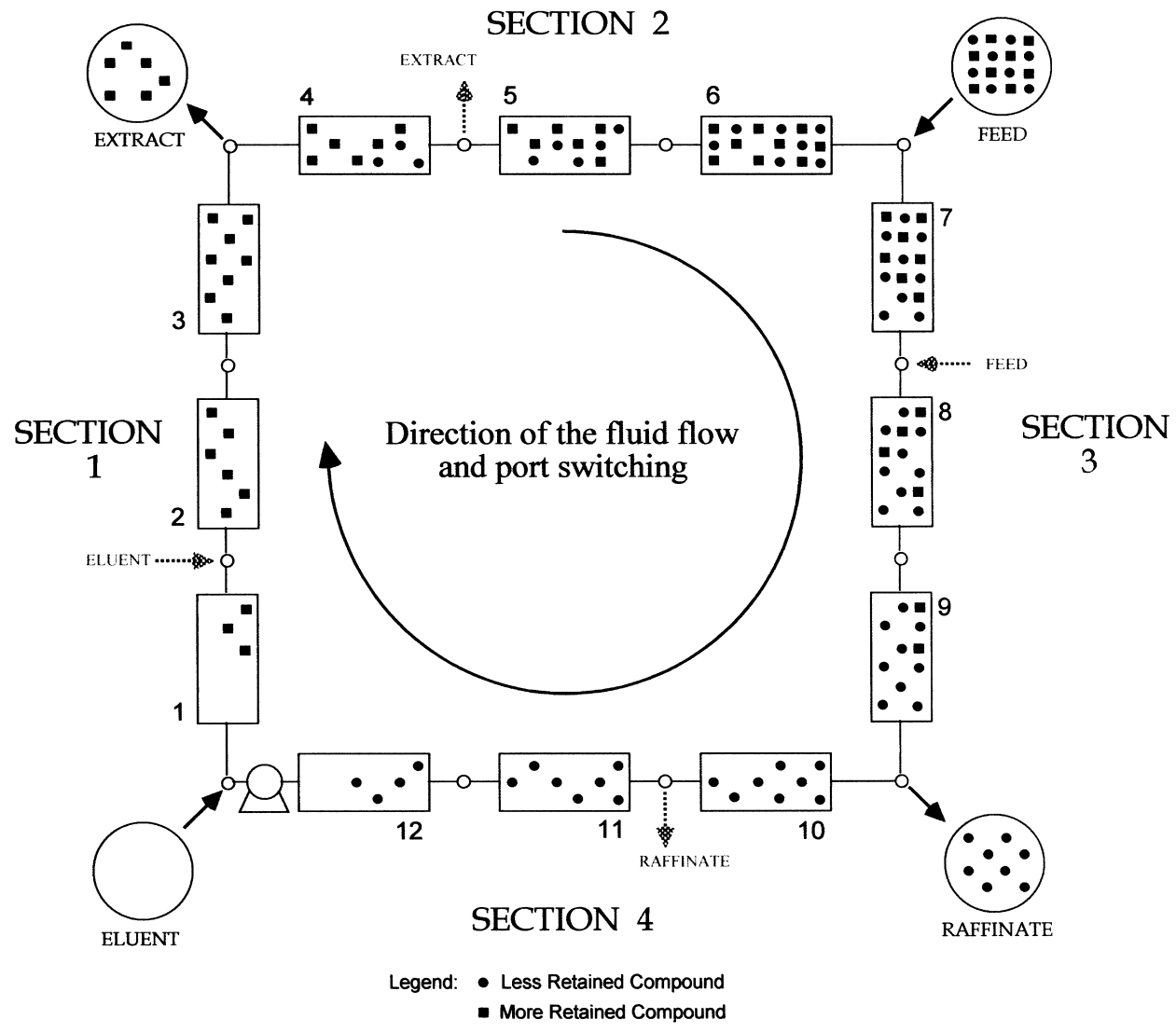

(b) TMB

solid circulation

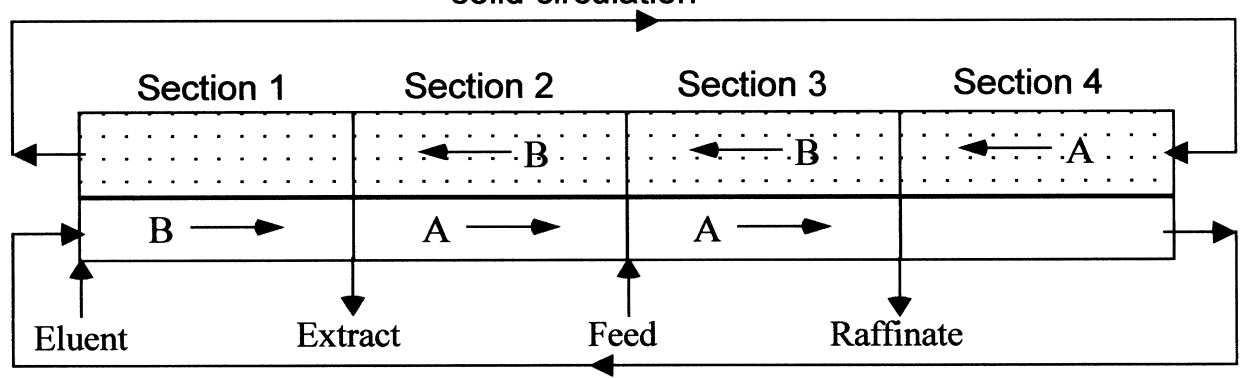

liquid recirculation

Fig. 1. Schematic diagram of: (a) a simulated moving bed (SMB); and (b) a true moving bed (TMB). 
Table 1

Transient SMB and TMB model equations

\section{Simulated Moving Bed model equations:}

Mass balance over a volume element of the bed $k$ :

$$
\begin{array}{r}
\frac{\partial C_{i k}}{\partial \theta}=\gamma_{k}^{*} \cdot\left\{\frac{1}{P e_{k}} \cdot \frac{\partial^{2} C_{i k}}{\partial x^{2}}-\frac{\partial C_{i k}}{\partial x}\right\} \\
-\frac{(1-\varepsilon)}{\varepsilon} \cdot \alpha_{k}\left(q_{i k}^{*}-q_{i k}\right)
\end{array}
$$

Mass balance in the particle:

$$
\frac{\partial q_{i k}}{\partial \theta}=\alpha_{k}\left(q_{i k}^{*}-q_{i k}\right) \quad(1 \mathrm{~b})
$$

Initial conditions :

$\theta=0: C_{i k}=q_{i k}=0$

Boundary conditions for column $\boldsymbol{k}$ :

$x=0: C_{i k}-\frac{1}{P e_{k}} \cdot \frac{\mathrm{d} C_{i k}}{\mathrm{~d} x}=C_{i k, 0}$

where $C_{i k, 0}$ is the inlet concentration of species $i$ in column $k$.

$x=1$ :

For a column inside a section and for extract and raffinate nodes:

$C_{i k}=C_{i k+1,0}$

For the eluent node:

$C_{i k}=\frac{\nu *}{\nu *} \cdot C_{i k+1,0}$

For the feed node:

$C_{i k}=\frac{\nu_{3}^{*}}{\nu \dddot{k}} C_{i k+1,0}-\frac{\nu_{\mathrm{F}}}{\nu \stackrel{2}{2}} C_{i}^{\mathrm{F}}$

\section{Global balances:}

Eluent node:

$\nu_{1}^{*}=\nu_{4}^{*}+\nu_{\mathrm{E}}$

Extract node:

$\nu_{2}^{*}=\nu_{1}^{*}-\nu_{\mathrm{X}}$

Feed node:

$\nu_{3}^{*}=\nu_{2}^{*}+\nu_{\mathrm{F}}$

Raffinate node:

$\nu_{4}^{*}=\nu_{3}^{*}-\nu_{\mathrm{R}}$

Multicomponent adsorption equilibrium isotherm: $q_{\mathrm{A} k}^{*}=f_{\mathrm{A}}\left(C_{\mathrm{A} k}, C_{\mathrm{B} k}\right)$

$q_{\mathrm{B} k}^{*}=f_{\mathrm{B}}\left(C_{\mathrm{A} k}, C_{\mathrm{B} k}\right)$
True Moving Bed model equations:

Mass balance over a volume element of the bed $j$ :

$\frac{\partial C_{i j}}{\partial \theta}=\gamma_{j} \cdot\left\{\frac{1}{P e_{j}} \cdot \frac{\partial^{2} C_{i j}}{\partial x^{2}}-\frac{\partial C_{i j}}{\partial x}\right\}$

$$
-\frac{(1-\varepsilon)}{\varepsilon} \cdot \alpha_{j}\left(q_{i j}^{*}-q_{i j}\right)
$$

Mass balance in the particle:

$\frac{\partial q_{i j}}{\partial \theta}=\frac{\partial q_{i j}}{\partial x}+\alpha_{j}\left(q_{i j}^{*}-q_{i j}\right)$

Initial conditions:

$\theta=0: C_{i j}=q_{i j}=0$

Boundary conditions for column $\boldsymbol{k}$ :

$x=0: C_{i j}-\frac{1}{P e_{j}} \cdot \frac{\mathrm{d} C_{i j}}{\mathrm{~d} x}=C_{i j, 0}$

where $C_{i j, 0}$ is the inlet concentration of species $i$ in section $j$.

$x=1$ :

For the eluent node:

$C_{i 4}=\frac{\nu_{1}}{\nu_{4}} \cdot C_{i 1,0}$

For the extract node:

$C_{i 1}=C_{i 2,0}$

For the feed node:

$C_{i 2}=\frac{\nu_{3}}{\nu_{2}} \cdot C_{i 3,0}-\frac{\nu_{\mathrm{F}}}{\nu_{2}} \cdot C_{i}^{\mathrm{F}}$

For the raffinate node:

$C_{i 3}=C_{i 4,0}$

and $q_{i 4}=q_{i 1,0}, q_{i 1}=q_{i 2,0}$

$q_{i 2}=q_{i 3,0}, q_{i 3}=q_{i 4,0}$

Global balances:

Eluent node:

$\nu_{1}=\nu_{4}+\nu_{\mathrm{E}}$

Extract node:

$\nu_{2}=\nu_{1}-\nu_{\mathrm{X}}$

Feed node:

$\nu_{3}=\nu_{2}+\nu_{\mathrm{F}}$

Raffinate node:

$\nu_{4}=\nu_{3}-\nu_{\mathrm{R}}$

Multicomponent adsorption equilibrium isotherm:

$q_{\mathrm{A} j}^{*}=f_{\mathrm{A}}\left(C_{\mathrm{A} j}, C_{\mathrm{B} j}\right)$

$q_{\mathrm{B} j}^{*}=f_{\mathrm{B}}\left(C_{\mathrm{A} j}, C_{\mathrm{B} j}\right)$ steady state achieved for the true countercurrent model.

In the TMB model, the solid phase is assumed to move in plug flow in the opposite direction to the fluid phase, while the inlet and outlet lines remain fixed. As a consequence, each column plays the same function, depending on its location. Model equations for the TMB model are equivalent to those presented for the SMB model, but include the term that represents the countercurrent movement of the solid phase. Model equations for the transient TMB model are also presented in Table 1, where the subscript $i$ refers to the component, and $j$ refers to the section. A more precise description of both models can be found elsewhere [33]. Model parameters are: the ratio between solid and fluid volumes, $(1-\varepsilon) / \varepsilon$; the ratio between fluid and solid interstitial velocities, $\gamma_{j}=v_{j} / u_{\mathrm{s}}$; the Peclet number, $P e_{j}=v_{j} L_{j} / D_{\mathrm{L} j}$; the 
number of mass transfer units, $\alpha_{j}=k L_{j} / u_{\mathrm{s}}$; and the adsorption equilibrium parameters. The equivalence between the TMB and the SMB models is made by keeping constant the liquid velocity relative to the solid velocity, that is, the liquid velocity in the SMB system is equal to the sum of liquid and solid velocities in the TMB model, $v_{j}^{*}=v_{j}+u_{\mathrm{s}}$. Also, the solid velocity in the TMB model must be evaluated from the value of the switch time interval $t^{*}$ of the SMB model, as $u_{\mathrm{s}}=L_{\mathrm{c}} / t^{*}$, where $L_{\mathrm{c}}$ is the length of one SMB column. This implies that the internal liquid flow-rates in both systems are not the same, but related by $Q_{j}^{*}=Q_{j}+\varepsilon V_{\mathrm{c}} / t^{*}$, where $Q_{j}^{*}$ and $Q_{j}$ are the internal liquid flow-rates in the SMB and TMB models, respectively, and $V_{\mathrm{c}}$ is the volume of one SMB column.

The SMB and TMB models, defined by a set of partial differential equations, were numerically solved by using PDECOL software [38] based on the method of orthogonal collocation in finite elements (OCFE). For the TMB model, there are four PDEs for each section: for each component there is a PDE resulting from the mass balance in a volume element of the bed, and another resulting from the mass balance in the particle. Since the TMB unit is composed of four sections, and considering a binary separation, the TMB system is defined by a set of 16 PDEs. In the SMB model, four equivalent PDEs must be written but now for each column. The four-section SMB system for a binary separation is then defined by a set of $4 N_{\mathrm{c}}$ PDEs, where $N_{\mathrm{c}}$ is the total number of columns used in the SMB unit. PDECOL software is based on the method of lines and uses a finite element collocation procedure for the discretization of the spatial variable. The collocation procedure reduces the PDE system to an initialvalue ODE system, which then depends only on the time variable. The time integration is then accomplished by using the ODE solver STIFIB, which is a modified version of the GEARIB ODE package developed by Hindmarsh [39].

\section{Results and discussion}

\subsection{System studied}

The chromatographic resolution of a racemic mixture of chiral epoxide enantiomers (Sandoz Pharma, Basel, Switzerland) was considered for simulation purposes. The chiral stationary phase used in this system is microcrystalline cellulose triacetate (Merck, Darmstadt, Germany) with an average particle diameter of $45 \mu \mathrm{m}$ and pure methanol is used as eluent. The adsorption equilibrium isotherms were measured at $25^{\circ} \mathrm{C}$ and are represented by a linear + Langmuir competitive model [18]:

$$
\begin{aligned}
& q_{\mathrm{A}}^{*}=1.35 C_{\mathrm{A}}+\frac{7.32 \cdot 0.087 C_{\mathrm{A}}}{1+0.087 C_{\mathrm{A}}+0.163 C_{\mathrm{B}}} \\
& q_{\mathrm{B}}^{*}=1.35 C_{\mathrm{B}}+\frac{7.32 \cdot 0.163 C_{\mathrm{B}}}{1+0.087 C_{\mathrm{A}}+0.163 C_{\mathrm{B}}}
\end{aligned}
$$

\subsection{SMB operation}

The first part of this work consists of the evaluation of the separation regions for different configurations and using the equivalent TMB model. A separation region is the area in a $\gamma_{3} \times \gamma_{2}$ plot where both extract and raffinate are pure $\left(\gamma_{2}\right.$ and $\gamma_{3}$ are the ratios between fluid and solid interstitial velocities in Sections 2 and 3, respectively). This plot, first proposed by Morbidelli and co-workers [40,41], is an important tool in the choice of the best operating conditions, providing that the constraints in Sections 1 and 4 are fulfilled, i.e. the flow-rate ratios in Sections 1 and 4, $\gamma_{1}$ and $\gamma_{4}$, are different from its critical values. It should be pointed out that the $\gamma_{j}$ ratios used in this work are related to the $m_{j}$ ratios used by Morbidelli and co-workers by:

$\gamma_{j}=\frac{\nu_{j}}{u_{\mathrm{s}}}=\frac{1-\varepsilon}{\varepsilon} \cdot m_{j}$

Fig. 2 shows a typical $\gamma_{3} \times \gamma_{2}$ plot that can be found in a non-linear TMB separation. Depending on the $\gamma_{2}$ and $\gamma_{3}$ values, we can find four regions: one where none of the extract and raffinate streams are pure, two regions where only one of the two streams is pure, and one region where both the extract and raffinate streams are pure. Of course, we should operate the system with operating conditions inside this last region, called the separation region, since the natural objective is to simultaneously obtain pure extract and raffinate streams. These separation regions, originally built by using the equilibrium theory model [41], can also be evaluated using more 


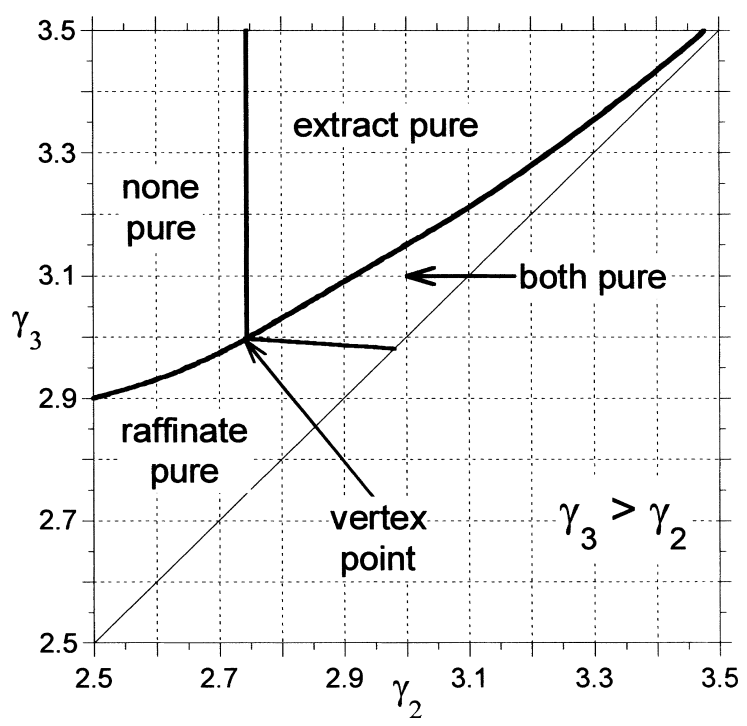

Fig. 2. Typical $\gamma_{3} \times \gamma_{2}$ plot for non-linear TMB operation.

precise models that take into account the resistances to mass transfer [12,18,42-44].

Table 2 presents the system characteristics and TMB model parameters. We considered a system constituted by a fixed total bed volume of $V_{\mathrm{T}}=420.5$ $\mathrm{ml}$ and fixed $\gamma_{1}$ and $\gamma_{4}$ parameters, very different from its critical values. Different SMB configurations were taken into consideration: an SMB system constituted by only one column per section (configuration 1111); two SMB systems constituted by five columns (configuration 1211, with two columns in section 2; and configuration 1121, with two columns in section 3); and an SMB unit with six columns and a configuration of 1221 (two columns in each central section). Table 3 presents the SMB configuration for each case and the correspondent TMB equivalence and model parameters. It should be pointed out that the length of each column depends on the number of columns used and is defined in such a way that the total bed volume and bed length are the same for all SMB configurations. The switch time interval also depends on the length of each SMB column and is evaluated in order to keep constant the equivalent solid velocity for all SMB configurations.

The results are presented in Fig. 3 and were obtained using the TMB model described in Table 1. A purity criterion of $99.9 \%$ was used for all configurations considered, which means that inside each separation region both extract and raffinate are, at least, $99.9 \%$ pure. Also shown is the separation region obtained considering the equilibrium theory, where axial dispersion and mass transfer resistances are neglected. The mass transfer coefficient used in this study was $k=0.4 \mathrm{~s}^{-1}$, which represents a situation where mass transfer resistance is not very important. Even in this situation, differences appear between the separation region obtained in this case and that predicted by the equilibrium theory. More pronounced differences would appear for lower values of mass transfer coefficient (mass transfer

Table 2

SMB unit characteristics and TMB model parameters

\begin{tabular}{lll}
\hline Total bed volume: & $V_{\mathrm{T}}=420.5 \mathrm{ml}$ & Solid $/$ fluid volumes: $(1-\varepsilon) / \varepsilon=1.5$ \\
Column diameter: & $D_{\mathrm{c}}=2.6 \mathrm{~cm}$ & Flow-rate ratios: \\
Total bed length: & $L_{\mathrm{T}}=79.2 \mathrm{~cm}$ & $\gamma_{1}=5.722$ \\
Feed concentration: & $C_{\mathrm{A}}^{\mathrm{F}}=C_{\mathrm{B}}^{\mathrm{F}}=5 \mathrm{~g} / 1$ & Mass transfer coefficient: $\quad k=0.4 \mathrm{~s}^{-1}$ \\
\hline
\end{tabular}

Table 3

SMB configurations, TMB equivalence and model parameters

\begin{tabular}{|c|c|c|c|c|c|c|c|}
\hline \multicolumn{4}{|c|}{ SMB configuration } & \multicolumn{4}{|c|}{ TMB equivalence and model parameters } \\
\hline $\begin{array}{l}\text { Number of } \\
\text { columns, } N_{\mathrm{c}}\end{array}$ & Configuration & $\begin{array}{l}\text { Column length, } \\
L_{\mathrm{c}}(\mathrm{cm})\end{array}$ & $\begin{array}{l}t^{*} \\
(\min )\end{array}$ & $\begin{array}{l}u_{\mathrm{s}}=L_{\mathrm{c}} / t^{*} \\
(\mathrm{~cm} / \mathrm{min})\end{array}$ & $\alpha_{\mathrm{c}}=k L_{\mathrm{c}} / u_{\mathrm{s}}$ & $\alpha_{\mathrm{T}}=N_{\mathrm{c}} \alpha_{\mathrm{c}}$ & $P e_{\mathrm{c}}$ \\
\hline 4 & 1111 & 19.80 & 6.60 & 3.00 & 158.40 & 633.6 & 2000 \\
\hline 5 & 1121 & 15.84 & 5.28 & 3.00 & 126.72 & 633.6 & 1600 \\
\hline 5 & 1211 & 15.84 & 5.28 & 3.00 & 126.72 & 633.6 & 1600 \\
\hline 6 & 1221 & 13.20 & 4.40 & 3.00 & 105.60 & 633.6 & 1333 \\
\hline
\end{tabular}




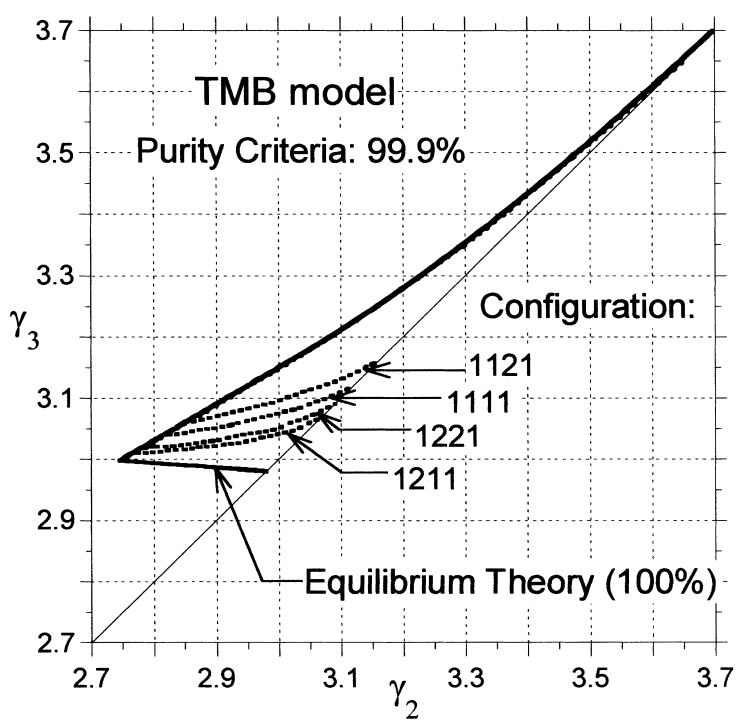

Fig. 3. $\gamma_{3} \times \gamma_{2}$ Separation regions for a $99.9 \%$ purity criterion using the TMB model and for the 1111, 1121, 1211, and 1221 configurations. Operating conditions and model parameters as in Tables 2 and 3. Also shown is the separation region considering equilibrium theory $(100 \%)$.

resistances more important), as we can find in a previous paper [18]. From Fig. 3 we conclude that, considering the TMB equivalence, the best results (larger separation regions) occur for the configurations that present a longer section 2 (between the extract and the feed nodes): in configuration 1211, section 2 is $40 \%$ of the total bed length; in configuration $1221,33.3 \%$; in configuration $1111,25 \%$; and in configuration 1121, 20\%. The dimensions of the separation regions obtained for the different configurations and shown in Fig. 3 are in agreement with this rule.

However, we may question if this is valid when considering the more realistic SMB model. In fact, we shall notice that SMB units with a low number of columns may have a steady state performance very different from that predicted by the TMB model. For instance, in contrast to the TMB model predictions presented in Fig. 3, there is no $99.9 \%$ separation region using the SMB model for the previous configurations. Fig. 4 compares the $99.0 \%$ separation regions considering the TMB and SMB models for the previous configurations: 1111, 1121, 1211 and 1221. This figure clearly shows the differences obtained with the two strategies in terms of steady state purities, and justifies the need to use the more precise SMB model when the unit has a low number of chromatographic columns. Of course these differences diminish with increment of the number of columns used and can be justified in a simple manner: the major differences between the TMB and SMB predictions occur for the 1111 and 1121 configurations (Fig. 4a and b) because only one column is used in section 2 , between the extract and feed nodes. In fact, as was noticed in a previous paper [33], for systems with only one column in section 2, the extract outlet will jump at the beginning of each switch time interval to the previous feed inlet, leading to a considerable decrease in the extract stream purity. The use of a second column in section 2 avoids this problem and drastically increases the separation region (see Fig. 4c for 1211 configuration), particularly the left region where, for the configurations with one simple column in section 2 , only raffinate was pure. On the other hand, we must notice that the increment of section 2 is made by decreasing the length of the other sections. Consequently, section 3 for the 1211 configuration (between the feed and the raffinate nodes) is shorter when compared with the two previous configurations (1111 and 1121) and we observe a diminishing of the upper zone of the separation region. For the 1211 configuration this upper zone is now characterized by a steady-state operation where only the extract stream is pure. The increment of this upper zone of the separation region can be obtained by using two columns in section 3, as we can confirm by looking at Fig. 4d. Using a 1221 configuration, the upper zone of the separation region is re-obtained without loosing the left zone, since we are also operating with two columns in section 2 .

Table 4 stresses these comparisons in terms of system productivity. The vertex point, defined as the point at the boundary of the separation region more distant from the diagonal $\gamma_{3}=\gamma_{2}$ (Fig. 2), represents the better operating conditions in terms of system productivity and solvent consumption. In fact, system productivity can be defined as the total amount of feed introduced in the system per volume of adsorbent bed and per unit of time, and is proportional to the difference $\left(\gamma_{3}-\gamma_{2}\right)$. Table 4 presents two comparison parameters: 

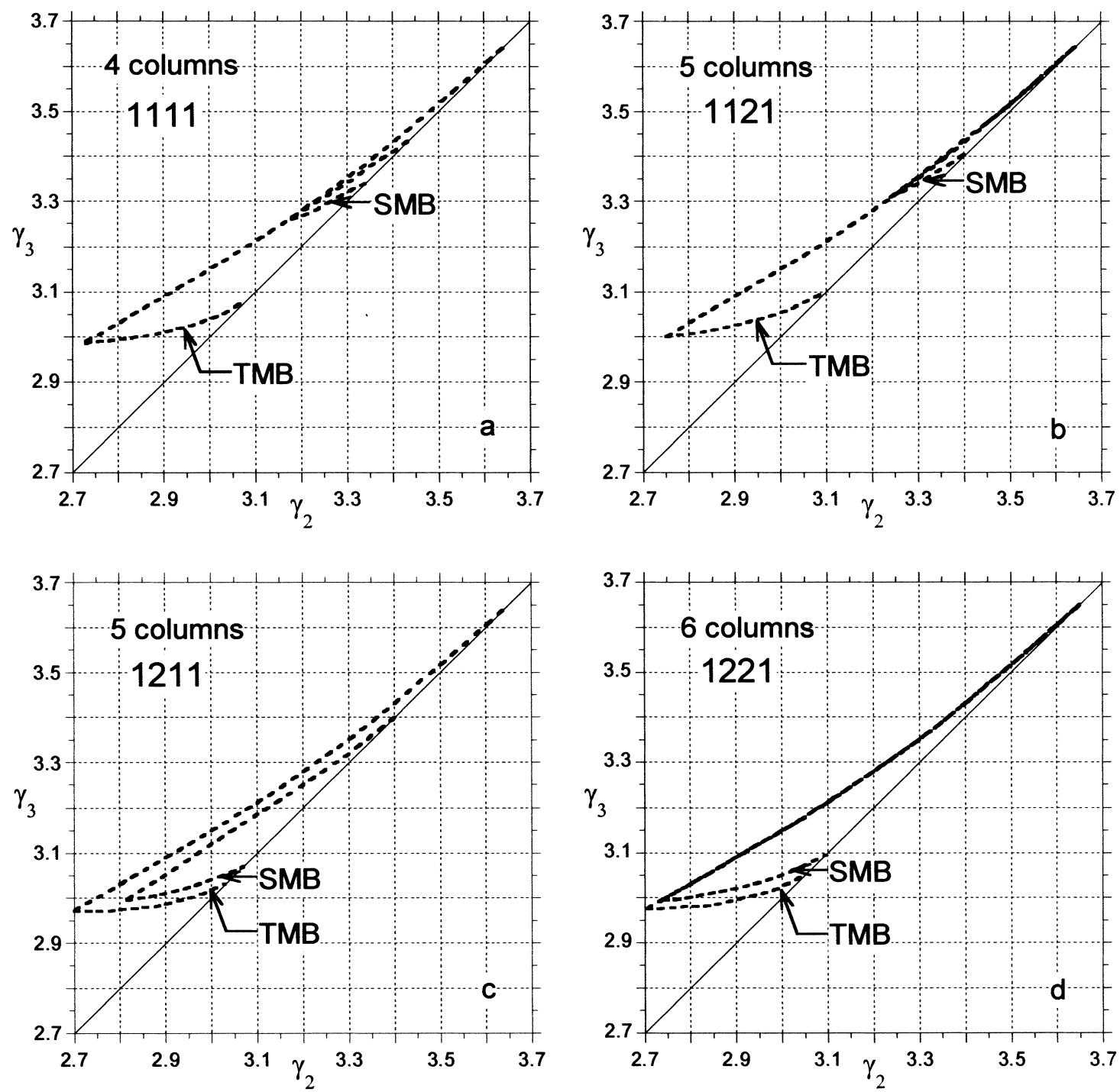

Fig. 4. $\gamma_{3} \times \gamma_{2}$ Separation regions for a $99.0 \%$ purity criterion. Comparison between TMB and SMB model results for the (a) 1111, (b) 1121, (c) 1211, and (d) 1221 configurations. Operating conditions and model parameters as in Tables 2 and 3.

Table 4

Comparison between TMB and SMB models in terms of system productivity (at the vertex point) for different configurations

\begin{tabular}{llll}
\hline Configuration & $\begin{array}{l}\text { Number of } \\
\text { columns, } N_{\mathrm{c}}\end{array}$ & $\pi^{\mathrm{SMB}}$ & $\eta=\frac{\pi^{\mathrm{SMB}}}{\pi^{\mathrm{TMB}}}$ \\
\hline 1111 & 4 & 0.085 & 0.333 \\
1121 & 5 & 0.070 & 0.280 \\
1211 & 5 & 0.175 & 0.648 \\
1221 & 6 & 0.255 & 0.944 \\
\hline
\end{tabular}

$$
\pi^{\mathrm{SMB}}=\left(\gamma_{3}-\gamma_{2}\right)^{\mathrm{SMB}}
$$

represents the difference $\left(\gamma_{3}-\gamma_{2}\right)$ obtained at the vertex point, considering the SMB model and, as stated before, is proportional to system productivity; and

$\eta=\frac{\pi^{\mathrm{SMB}}}{\pi^{\mathrm{TMB}}}=\frac{\left(\gamma_{3}-\gamma_{2}\right)^{\mathrm{SMB}}}{\left(\gamma_{3}-\gamma_{2}\right)^{\mathrm{TMB}}}$

represents the ratio between the differences $\left(\gamma_{3}-\gamma_{2}\right)$ 
obtained at the vertex points using the SMB and TMB models and somehow quantifies the differences between the two strategies of modeling for a specific configuration. For instance, for the 1111 configuration, the system productivity at the vertex point predicted by the more realistic SMB model is only $33.3 \%$ of that indicated by the ideal TMB model. For the 1221 configuration, this difference is reduced, with the SMB system productivity being $94.4 \%$ of that predicted by the TMB operation. It must be noticed, however, that the SMB model can be useful for SMBs with a higher subdivision of the adsorbent bed. Even in these situations, the configuration used affects the separation performance of the unit, as was recently reported by Silva et al. for an SMB system constituted by 12 chromatographic columns [45].

\subsection{Varicol operation}

As was presented in the Introduction, Varicol is a new continuous chromatographic process and consists of a modification of the classic SMB operation by introducing a non-synchronous shift of the inlet and outlet lines. Fig. 5 shows an illustrative example of Varicol operation and compares it to the classic SMB system. Consider an SMB system with a 1211 configuration (Fig. 5, left). At the beginning of each switch time interval, all inlet and outlet streams will jump one column forward, in the direction of the liquid phase. This jump is synchronous, so, for this example, sections 1,3 and 4 will have always one column, while section 2 will have always two. Consider now an SMB 1121 configuration: in this case, the feed inlet is at the beginning of the third column (Fig. 5, upper right). At half of the switch time interval, the feed inlet will jump to the beginning of the fourth column, while all the other lines (eluent, extract, and raffinate) remain fixed (Fig. 5,

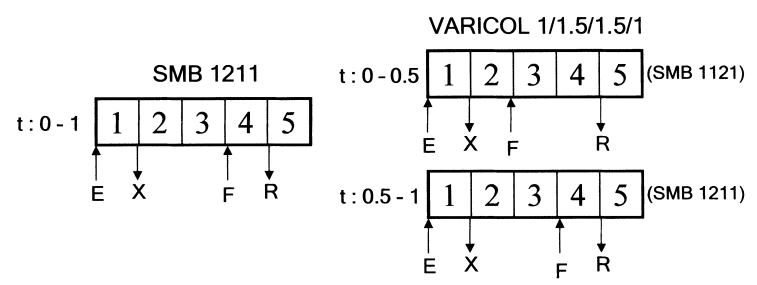

Fig. 5. Example of a Varicol operation: five-column SMB 1211 (left) and five-column Varicol 1/1.5/1.5/1 configurations (right). lower right). From now on, the configuration is not 1121 but 1211, and will stay like this until the end of the switch time interval is reached. We may say that during the entire switch time interval we have two different SMB configurations: in the first half, the SMB 1121 configuration; and in the last, the SMB 1211 configuration. This simple example represents a Varicol operation, since the system is operating with a number of columns per section that is not constant in time. For this example, sections 1 and 4 always have one column; however, sections 2 and 3 have one column during one half of each switch time interval and two columns during the other half. Averaging, we may say that the system described before has one column in sections 1 and 4 , and one and a half columns in sections 2 and 3 ; i.e. we are in the presence of a Varicol 1/1.5/1.5/1 configuration.

The Varicol process was proposed by Novasep and the first experimental results were recently published [46]. The last part of this work will present an effective comparison between SMB and Varicol operations, by evaluating the $\gamma_{3} \times \gamma_{2}$ separation regions for both cases. Model equations to simulate the Varicol process are the same as used in the SMB model, but must take into account the non-synchronous shift of the inlet and outlet lines.

Fig. 6 presents in the same plot the separation regions obtained for the better five-column SMB configuration (SMB 1211), the better six-column SMB configuration (SMB 1221), and the five-column Varicol 1/1.5/1.5/1 configuration. This figure clearly shows that this five-column Varicol configuration presents a separation region closer to that obtained for the better six-column SMB than to that obtained for the better five-column SMB operation. Table 5 also shows that this result is valid in terms of system productivity. This example shows the potential of the Varicol operation using a low number of chromatographic columns, since a five-column Varicol process presents a performance close to the better six-column SMB operation. Moreover, the five-column Varicol configuration can still be optimized. It should be pointed out that the Varicol process allows an unlimited number of configurations. Fig. 7 presents the values obtained for the difference $\left(\gamma_{3}-\gamma_{2}\right)$ at the vertex point of the separation regions obtained for other five-column Varicol configurations. In this work, we limited our study by 


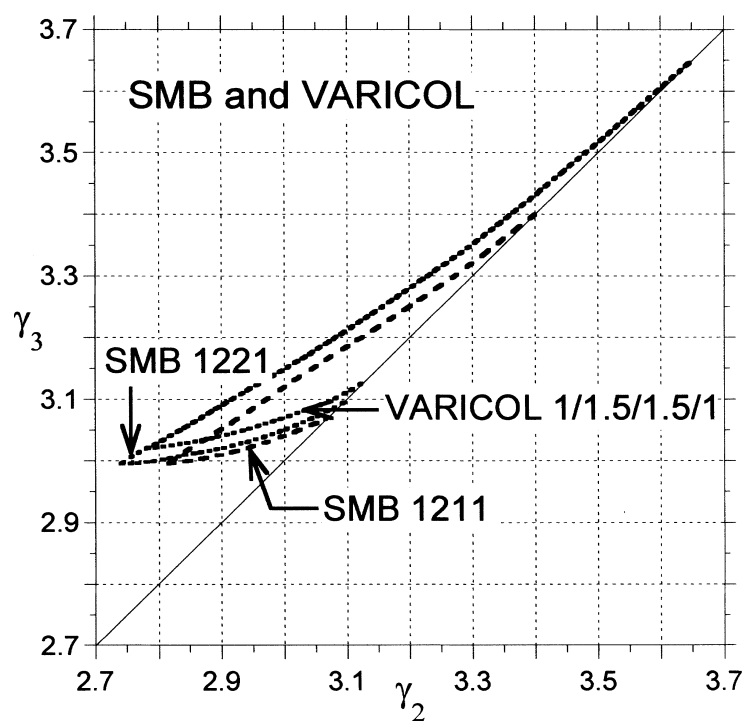

Fig. 6. Comparison between the better five-column SMB configuration (SMB 1211), the better six-column SMB configuration (SMB 1221), and the five-column Varicol 1/1.5/1.5/1 configuration, for a $99.0 \%$ purity criterion, in terms of $\gamma_{3} \times \gamma_{2}$ separation regions.

\section{Table 5}

Comparison between the better five- and six-column SMB configurations (SMB 1211 and SMB 1221), and the five-column Varicol $1 / 1.5 / 1.5 / 1$ configuration, for a $99.0 \%$ purity criterion, in terms of system productivity at the vertex point

\begin{tabular}{llc}
\hline Configuration & $\begin{array}{l}\text { Number of } \\
\text { columns, } N_{\mathrm{c}}\end{array}$ & $\pi^{\mathrm{SMB}}$ \\
\hline SMB 1211 & 5 & 0.175 \\
Varicol 1/1.5/1.5/1 & 5 & 0.240 \\
SMB 1221 & 6 & 0.255 \\
\hline
\end{tabular}

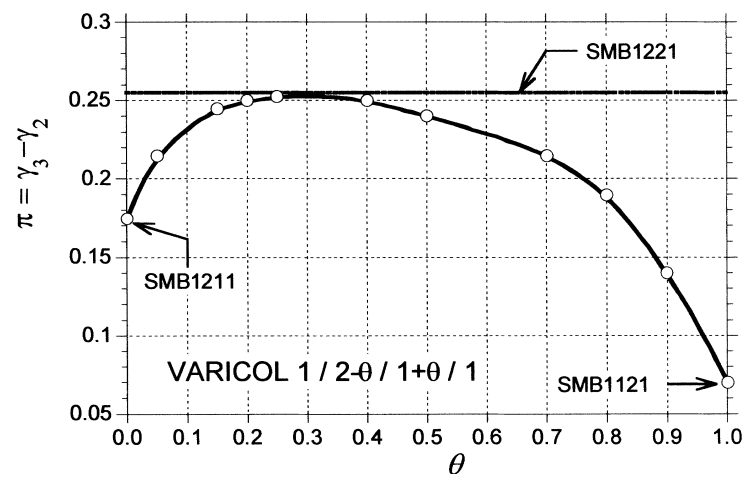

Fig. 7. System productivity for a five-column Varicol $1 / 2-\theta / 1+$ $\theta / 1$ configuration, with $0 \leq \theta \leq 1$. using always one column in sections 1 and 4, but different cases were evaluated for the average number of columns in sections 2 and 3. Fig. 7 presents the results obtained for the five-column Varicol 1/ $2-\theta / 1+\theta / 1$ configuration, with $0 \leq \theta \leq 1$ : if $\theta=$ 0 , we are in the presence of the SMB 1211 configuration; if $\theta=1$, the SMB 1121 SMB configuration; all the other situations represent new possibilities not allowed in the classic SMB operation. In this particular case, the five-column Varicol 1/1.7/1.3/1 configuration $(\theta=0.3)$ presents the same productivity obtained by the better six-column SMB configuration. As a final remark, it should be pointed out that a complete optimization of the Varicol process should take into account all four sections and not only the two central Sections, 2 and 3. Consequently, further improvements in the Varicol system productivity can be obtained, as was shown in two recent publications $[47,48]$.

\section{Conclusions}

SMB chromatography is a well-established technique and the number of industrial applications is increasing, particularly for chiral chromatographic resolution of enantiomeric species. These units generally use a low number of chromatographic columns. In this situation, evaluation of the SMB operating conditions avoids the use of the ideal countercurrent TMB model, and a more realistic SMB model, which takes into account the periodic shift of the injection and collection points, is needed. For an effective comparison between the predictions given by the two strategies of modeling, the $\gamma_{3} \times$ $\gamma_{2}$ separation regions can be evaluated using the TMB and SMB models. The differences obtained by the two models depend particularly on the number of columns used and its configuration.

The use of SMB units with a low number of chromatographic columns presents the advantage of a simpler and more economic solution, but may lead to a significant loss of process purity or system productivity. The Varicol process, a modification of the classic SMB operation by introducing a nonsynchronous shift of the inlet and outlet lines, can overcome this disadvantage and present performances closer to those obtained with SMB units with a 
higher subdivision of the adsorbent bed. The modeling and simulation tools defined in this work can also be used to evaluate the $\gamma_{3} \times \gamma_{2}$ separation regions for Varicol processes, in order to compare its performance with the classical SMB operation and to study the influence of the number of columns and its configuration on the system performance.

\section{Nomenclature}

Symbols

$C \quad$ Fluid phase concentration $(\mathrm{g} / \mathrm{l})$

$D_{\mathrm{L}} \quad$ Axial dispersion coefficient $\left(\mathrm{cm}^{2} / \mathrm{s}\right)$

$D_{\text {c }} \quad$ Diameter of an SMB column $(\mathrm{cm})$

$k^{c} \quad$ Mass transfer coefficient $\left(\mathrm{s}^{-1}\right)$

$L_{\mathrm{c}} \quad$ Length of an SMB column $(\mathrm{cm})$

$L_{j} \quad$ Length of a TMB section $(\mathrm{cm})$

$L_{\mathrm{T}} \quad$ Total bed length $(\mathrm{cm})$

$N_{\mathrm{c}} \quad$ Number of columns

$\mathrm{Pe} \quad$ Peclet number

$Q \quad$ Volumetric liquid flow-rate in TMB operation $(\mathrm{ml} / \mathrm{min})$

$Q^{*} \quad$ Volumetric liquid flow-rate in SMB operation $(\mathrm{ml} / \mathrm{min})$

$q \quad$ Average adsorbed phase concentration $\left(\mathrm{g} / 1_{\text {particle }}\right)$

$q^{*} \quad$ Adsorbed phase concentration in equilibrium with $C\left(\mathrm{~g} / \mathrm{l}_{\text {particle }}\right)$

$t^{*} \quad$ Switch time interval (s)

$u_{\mathrm{s}} \quad$ Interstitial solid velocity in the TMB operation $(\mathrm{cm} / \mathrm{s})$

$V_{\mathrm{c}} \quad$ Volume of an SMB column $(\mathrm{ml})$

$V_{\mathrm{T}} \quad$ Total bed volume (ml)

$v \quad$ Interstitial fluid velocity in the TMB operation $(\mathrm{cm} / \mathrm{s})$

$v^{*} \quad$ Interstitial fluid velocity in the SMB operation $(\mathrm{cm} / \mathrm{s})$

$x \quad$ Dimensionless axial coordinate

Greek symbols

$\alpha \quad$ Number of mass transfer units

$\gamma \quad$ Ratio between fluid and solid interstitial

velocities in TMB operation

$\gamma^{*} \quad$ Ratio between fluid and solid interstitial velocities in SMB operation

$\varepsilon \quad$ Bed porosity $\eta \quad$ Comparison parameter, defined as in Eq. (16) in Table 1

$\theta \quad$ Dimensionless time

$\pi \quad$ System productivity parameter, defined as in Eq. (15) in Table 1

\section{Subscripts}

A Less retained component

B More retained component

E Eluent

F $\quad$ Feed

$\mathrm{R} \quad$ Raffinate

X Extract

$i \quad$ Component index $(i=\mathrm{A}, \mathrm{B})$

$j \quad$ Section index $(j=1,2,3,4)$

$k \quad$ Column index $\left(k=1, \ldots, N_{\mathrm{c}}\right)$

\section{References}

[1] G. Fuchs, R.M. Nicoud, M. Bailly, in: M. Perrut (Ed.), Proceedings of the 9th International Symposium on Preparative and Industrial Chromatography, Société Française de Chemie, Nancy, 1992, p. 395.

[2] M. Negawa, F. Shoji, J. Chromatogr. 590 (1992) 113.

[3] R.M. Nicoud, G. Fuchs, P. Adam, M. Bailly, E. Küsters, F. Antia, R. Reuille, E. Schmid, Chirality 5 (1993) 267.

[4] R.M. Nicoud, M. Bailly, J.N. Kinkel, R.M. Devant, T. Hampe, E. Küsters, in: R.M. Nicoud (Ed.), Simulated Moving Bed: Basics and Applications, INPL, Nancy, 1993, p. 65.

[5] C.B. Ching, B.G. Lim, E. Lee, S.C. Ng, J. Chromatogr. 634 (1993) 215.

[6] E. Küsters, G. Gerber, F.D. Antia, Chromatographia 40 (1995) 387.

[7] F. Charton, R.M. Nicoud, J. Chromatogr. A 702 (1995) 97.

[8] B.G. Lim, C.B. Ching, J. Chromatogr. A 734 (1996) 247.

[9] H.W. Dandekar, A.K. Chandhok, J.W. Priegnitz, in: M.D. LeVan (Ed.), Fundamentals of Adsorption, Kluwer, Boston, MA, 1996, p. 243.

[10] L.S. Pais, J.M. Loureiro, A.E. Rodrigues, Chem. Eng. Sci. 52 (1997) 245.

[11] D.W. Guest, J. Chromatogr. A 760 (1997) 157.

[12] L.S. Pais, J.M. Loureiro, A.E. Rodrigues, J. Chromatogr. A 769 (1997) 25.

[13] E. Cavoy, M.F. Deltent, S. Lehoucq, D. Miggiano, J. Chromatogr. A 769 (1997) 49.

[14] M. Schulte, R. Ditz, R.M. Devant, J.N. Kinkel, F. Charton, J. Chromatogr. A 769 (1997) 93.

[15] E. Francotte, P. Richert, J. Chromatogr. A 769 (1997) 101.

[16] E. Francotte, P. Richert, M. Mazzotti, M. Morbidelli, J. Chromatogr. A 796 (1998) 239. 
[17] C. Heuer, E. Küsters, T. Plattner, A. Seidel-Morgenstern, J. Chromatogr. A 827 (1998) 175.

[18] L.S. Pais, J.M. Loureiro, A.E. Rodrigues, J. Chromatogr. A 827 (1998) 215.

[19] S. Nagamatsu, K. Murazumi, S. Makino, J. Chromatogr. A 832 (1999) 55.

[20] M. Pedeferri, G. Zenoni, M. Mazzotti, M. Morbidelli, Chem. Eng. Sci. 54 (1999) 3735.

[21] L.S. Pais, J.M. Loureiro, A.E. Rodrigues, Sep. Purif. Technol. 20 (2000) 67.

[22] S. Lehoucq, D. Verhève, A.V. Wouwer, E. Cavoy, AIChE J. 46 (2000) 247.

[23] E. Küsters, C. Heuer, D. Wieckhusen, J. Chromatogr. A 874 (2000) 155.

[24] S. Khattabi, D.E. Cherrak, K. Mihlbacher, G. Guiochon, J. Chromatogr. A 893 (2000) 307.

[25] M. Schulte, J. Strube, J. Chromatogr. A 906 (2001) 399.

[26] E. Huthmann, M. Juza, J. Chromatogr. A 908 (2001) 185.

[27] M. Schulte, R. Devant, R. Grosser, J. Pharm. Biomed. Anal. 27 (2002) 627.

[28] C. Migliorini, M. Mazzotti, G. Zenoni, M. Morbidelli, AIChE J. 48 (2002) 69.

[29] R.M. Nicoud, Pharm. Tech. Europe 11 (No. 3) (1999) 36.

[30] R.M. Nicoud, Pharm. Tech. Europe 11 (No. 4) (1999) 28.

[31] M. McCoy, Chem. Eng. News 19 (2000) 17.

[32] S.C. Stinson, Chem. Eng. News 14 (2001) 45.

[33] L.S. Pais, J.M. Loureiro, A.E. Rodrigues, AIChE J. 44 (1998) 561.

[34] R.M. Nicoud, R.E. Majors, LC·GC Eur. 13 (2000) 887.
[35] J. Blehault, W. Hauck, R.M. Nicoud, Seminar on Batch and SMB Design and Applications, AIChE Meeting, Chicago, IL, March 2001

[36] P. Adam, R.M. Nicoud, M. Bailly, O. Ludemann-Hombourger, US Patent 6136198 (2000).

[37] O. Ludemann-Hombourger, R.M. Nicoud, M. Bailly, Sep. Sci. Technol. 35 (2000) 1829.

[38] N. Madsen, R. Sincovec, ACM Trans. Math. 5 (1979) 326.

[39] A. Hindmarsh, Rep. UCID-30130, Lawrence Livermore Laboratory, Livermore, 1976.

[40] G. Storti, M. Mazzotti, M. Morbidelli, S. Carrà, AIChE J. 39 (1993) 471.

[41] M. Mazzotti, G. Storti, M. Morbidelli, J. Chromatogr. A 769 (1997) 3.

[42] D. Azevedo, L.S. Pais, A.E. Rodrigues, J. Chromatogr. A 865 (1999) 187.

[43] C. Migliorini, A. Gentilini, M. Mazzotti, M. Morbidelli, Ind. Eng. Chem. Res. 38 (1999) 2400.

[44] G. Biressi, O. Ludemann-Hombourger, M. Mazzotti, R.M. Nicoud, M. Morbidelli, J. Chromatogr. A 876 (2000) 3.

[45] E.B. Silva, A.U. Souza, S.U. Souza, Sep. Sci. Technol. 37 (2002) 1489.

[46] O. Ludemann-Hombourger, G. Pigorini, R.M. Nicoud, D.S. Ross, G. Terfloth, J. Chromatogr. A 947 (2002) 59.

[47] A. Toumi, F. Hanisch, S. Engell, Ind. Eng. Chem. Res. 41 (2002) 4328

[48] Z. Zhang, K. Hidajat, A. Ray, M. Morbidelli, AIChE J. 48 (2002) 2800. 